\title{
Chloroquine resistant falciparum malaria among security forces personnel in the Northern Province of Sri Lanka
}

HAC Hapuarachchi' ${ }^{1}$, MYD Dayanath ${ }^{2}$, S Abeysundara ${ }^{3}$, KBAT Bandara $^{3}$, W Abeyewickreme ${ }^{4}$ and N R de Silva ${ }^{5}$

(Index words: drug resistance, Plasmodium falciparum, Plasmodium vivax, soldiers)

\begin{abstract}
Objective To determine the occurrence and species distribution of malaria and the extent of chloroquine resistance among security forces personnel in a selected region of the Northern Province of Sri Lanka.
\end{abstract}

Design A descriptive study.

Setting Mannar District in the Northern Province.

Methods Nine hundred and seventy five security personnel were screened for malaria by microscopy. Those who were positive were treated with chloroquine and were subjected to 28 day in vivo assay to determine chloroquine resistance. In vitro microtest assay was performed to determine the response of Plasmodium falciparum isolates to chloroquine in vitro.

Results Of the 975 personnel screened, 181 (18.6\%) were positive for malaria. $P$. falciparum was the predominant species $(n=125 ; 69.1 \%)$. The rest were due to $P$. vivax $(n=42 ; 23.2 \%)$ and mixed infections $(n=14 ; 7.7 \%)$. This was an inversion of the usual species distribution pattern in the country. In vivo assay revealed 38 (53.5\%) $P$. falciparum infections as chloroquine resistant. Fifteen of $23(65.2 \%) P$. falciparum isolates showed evidence of resistance in vitro. None of the $P$. vivax infections showed evidence of chloroquine resistance. There was no significant difference in the severity of clinical disease between chloroquine resistant and sensitive infections at first presentation. Recrudescent $P$. falciparum infections had significantly lower mean parasite densities as well as lower clinical scores at recrudescence than at first presentation.

Conclusion Results demonstrate the high prevalence of malaria and chloroquine resistance in the study area and explains several contributory factors for this. There is an urgent need to review antimalarial drug policies in Sri Lanka.

\section{Introduction}

Chloroquine still remains the first-line drug for treatment of uncomplicated malaria in Sri Lanka. Sulfadoxinepyrimethamine (S-P) is used as the main second-line drug.
Resistance of $P$. falciparum to both drugs has been reported in the country. Chloroquine resistance was first reported in 1984 [1] and its spread has been identified by the national malaria control programme $[2,3]$ as an important factor influencing the malaria transmission pattern in the country. The true extent of chloroquine resistance is not known, but one study found that $30 \%$ to $55 \%$ of $P$. falciparum isolates from both endemic and non-endemic areas showed some degree of resistance [4]. Several cases of S-P resistance have been reported [5,6], but it is not yet considered a widespread problem.

Much of the morbidity and mortality due to malaria in Sri Lanka now occurs in the Northern and Eastern Provinces, which have been affected by armed conflict since the early 1980 s. More than $60 \%$ of new cases and more than $90 \%$ of deaths due to malaria reported in 2001 were from these two provinces [3]. Malaria surveillance and control activities have been virtually paralysed for more than 20 years in these areas, and published annual figures probably underestimate the true prevalence. Apart from the local inhabitants, thousands of security forces personnel have been stationed in the Northern Province for the last 20 years. Many are from non-endemic areas, and therefore, at a greater risk of contracting malaria, and carrying it to other parts of the country.

Our study was done to determine the malarial species distribution and the efficacy of chloroquine as the firstline treatment for uncomplicated malaria in security forces personnel in a selected region of the Northern Province of Sri Lanka.

\section{Materials and methods \\ Study site and population}

This study was carried out in an operational area situated in the Mannar district, about $28 \mathrm{~km}$ east of Mannar on the main supply route to Vavuniya (Figure 1). The population consisted of Sri Lankan armed forces and Police personnel.

\footnotetext{
${ }^{1}$ Lecturer, ${ }^{2}$ Technical Assistant, ${ }^{3}$ Technical Officers, ${ }^{4}$ Senior Lecturer, ${ }^{5}$ Professor and Head, Department of Parasitology, Faculty of Medicine, University of Kelaniya, P. O. Box 6, Thalagolla Road, Ragama.

Correspondence: HACH, Faculty of Medicine, University of Kelaniya, P.O. Box 6, Thalagolla Road, Ragama, Sri Lanka. Tel / Fax: 0094112 953412, e-mail: <chandith@lycos.com> (Competing interests: none declared). Received 30 November 2003 and accepted 18 January 2004.
} 
Figure 1. Map of Sri Lanka showing the main supply route from Vavuniya to Mannar (๑-indicates the study area).

\section{Study participants and protocol}

Patients with any symptoms suggestive of acute malaria were screened by examining a thick blood smear stained with $2 \%$ Giemsa. At least 100 fields were examined before a patient was confirmed as negative. Patient enrolment was based on the following criteria: (a) an elevated axillary temperature of or more than $37.5^{\circ} \mathrm{C}$ at presentation or a history of fever in last 24 hours, (b) mono-infection of $P$. falciparum or $P$. vivax with $>250$ asexual parasites per microliter, (c) absence of severe malaria [7], (d) absence of a history of allergy to either chloroquine or S-P and absence of a history of G6PD deficiency, and (e) willingness to provide informed consent to participate in the study.

On enrolment each patient was examined and evaluated by a medical officer for symptoms, duration of the illness, antimalarial therapy during the last four weeks, previous malaria episodes, duration of stay in this area and axillary temperature. Information was recorded in a pre-tested questionnaire. The severity of each infection was determined using a previously validated clinical scoring system [8]. Five $\mathrm{ml}$ of venous blood was collected from each on the day of enrolment (day 0) using a sterile disposable syringe. Parasite density was calculated by counting the number of asexual parasites/200 white blood cells (WBC) in a thick film assuming an average WBC of $8000 / \mu \mathrm{L}$ of blood. The Dill-Glazko test was performed on a urine sample from each patient to detect urinary 4-aminoquinolones [9].

\section{Treatment and follow up}

All positive patients were treated with chloroquine as recommended by the national Anti-Malaria Campaign, $600 \mathrm{mg}$ on day $1,600 \mathrm{mg}$ on day 2 and $300 \mathrm{mg}$ on day 3 . Primaquine was also given as recommended for $P$. vivax and P. falciparum, i.e. $7.5 \mathrm{mg}$ twice a day for 14 days and a $45 \mathrm{mg}$ single dose. In addition, paracetamol was administered to all febrile patients. Enrolled patients were reviewed on days $1,2,7,14,21$ and 28. During each follow up visit, patients were reassessed clinically and parasitologically.

The parasitological response was determined using modified WHO criteria [10]. If the reappearance took place on days 8 to 14 , it was defined as an early recrudescence, and as a late recrudescence if it was between days 15 to 28. Those who showed evidence of any form of chloroquine resistance were treated with the recommended single dose of $1500 \mathrm{mg}+75 \mathrm{mg} \mathrm{S}-\mathrm{P}$. All drugs were administered under supervision and re-administered if the patient vomited within $30 \mathrm{~min}$.

\section{In vitro assessment of parasite response to chloroquine}

Patients were selected for this assay according to the guidelines given by WHO [11]. Chloroquine resistance of $P$. falciparum was evaluated using in vitro micro-test plates provided by the Vector Control Research Unit, Malaysia. At least $10 \%$ of schizont maturation in the control well was considered as the criterion for a successful test. An isolate was considered to be chloroquine resistant if it showed schizont maturation at or beyond 8 pmol concentration of chloroquine [11].

Approval for this study was obtained from the Ethics Committee of the Faculty of Medicine, University of Kelaniya. Data analysis was done using independent $t$ test for two means and Fisher's exact test. Log transformations were used for positively skewed distributions and comparison was done using the independent $t$ test for logtransformed data. $\mathrm{P}$ value less than 0.05 was considered as significant.

\section{Results}

In all, 975 patients were screened during the period January-June 2002; 181 (18.6\%) were found positive for malarial parasites. Of these, $125(69.1 \%)$ had $P$. falciparum, 42 (23.2\%) had $P$. vivax, and 14 (7.7\%) had mixed infections. From those who were malaria positive, 137 (75.7\%) patients, including 102 P. falciparum and $35 P$. vivax infections were enrolled for the study. All were men.

Of the total number enrolled, 94 (68.6\%) completed the 28 days follow up period. This included 71 with 
$P$. falciparum and 23 with $P$ vivax infections. Of the P. falciparum infections that completed follow up, 38 $(53.5 \%)$ showed evidence of chloroquine resistance. Thirty three $(86.8 \%)$ of these belonged to the R1 group and five (13.2\%) showed R11 resistance. Of R1 group, $36.4 \%$ recrudesced early, implying that chloroquine resistance is increasing in severity. None of the $P$. falciparum infections showed R111 grade resistance. None of the $P$. vivax infections showed evidence of chloroquine resistance.

Table 1 summarises the characteristics of patients with chloroquine resistant and sensitive infections in vivo. The geometric mean of parasite density $(5376.7 / \mu \mathrm{L})$ and mean axillary temperature of chloroquine resistant $P$. falciparum primary infections were significantly higher than the chloroquine sensitive infections $(2339 / \mu \mathrm{L})$ $(\mathrm{t}=2.050, \mathrm{p}<0.05,95 \%$ CI $1-5.25$, and $\mathrm{t}=3.57, \mathrm{p}<0.001$, 95\% CI 0.44-1.56). There were no significant differences between the mean clinical scores (16.5 and 13.7) and geometric mean of number of previous malaria attacks (1.94 and 2.9), and duration of stay in the area (7.6 and 13.5 months).

Table 1. Comparison of patients with chloroquine resistant and chloroquine sensitive $P$. falciparum infections

\begin{tabular}{|c|c|c|}
\hline Characteristic & $\begin{array}{l}C Q \text { resistant } \\
(n=38)\end{array}$ & $\begin{array}{l}C Q \text { sensitive } \\
(n=33)\end{array}$ \\
\hline $\begin{array}{l}\text { Geometric mean of } \\
\text { duration of stay in } \\
\text { this area (months) }\end{array}$ & $\begin{array}{l}7.6(\text { s.d. of } \log s= \\
0.57, r=1-60)\end{array}$ & $\begin{array}{l}13.5(\mathrm{~s} . \mathrm{d} \text { of } \\
\operatorname{logs}=0.54, \\
r=1-60)\end{array}$ \\
\hline $\begin{array}{l}\text { Geometric mean of } \\
\text { number of past } \\
\text { malaria infections }\end{array}$ & $\begin{array}{l}1.94 \text { (s.d. of } \log s= \\
0.37, r=0-15)\end{array}$ & $\begin{array}{c}2.9 \text { (s.d. of } \\
\operatorname{logs}=0.49, \\
r=0-37)\end{array}$ \\
\hline $\begin{array}{l}\text { Mean initial } \\
\text { temperature }\left({ }^{0} \mathrm{~F}\right)\end{array}$ & $\begin{array}{l}101.3(\text { s.d. }=1.237 \\
r=99.6-104)\end{array}$ & $\begin{array}{l}100.4 \\
\text { s.d.=1.071, } \\
r=99.5-104)\end{array}$ \\
\hline $\begin{array}{l}\text { Mean temperature at } \\
\text { reappearance of } \\
\text { parasitaemia }\left({ }^{\circ} \mathrm{F}\right)\end{array}$ & $\begin{array}{l}99.6 \text { (s.d. }=1.201 \text {, } \\
99.5-102.3)\end{array}$ & \\
\hline $\begin{array}{l}\text { Mean initial clinical } \\
\text { score }\end{array}$ & $\begin{array}{l}16.5 \text { (s.d. }=6.705 \\
r=2-30)\end{array}$ & $\begin{array}{l}13.7 \text { (s.d. }=7.191, \\
r=1-27)\end{array}$ \\
\hline $\begin{array}{l}\text { Mean clinical score } \\
\text { at reappearance of } \\
\text { parasitaemia }\end{array}$ & $\begin{array}{l}6.6 \text { (s.d. }=7.394 \\
r=0-30)\end{array}$ & \\
\hline $\begin{array}{l}\text { Geometric mean } \\
\text { of initial parasite } \\
\text { density }(\text { per } \mu \mathrm{L})\end{array}$ & $\begin{array}{l}5376.7 \text { (s.d. of } \\
\operatorname{logs}=3.73, \\
r=320-132000)\end{array}$ & $\begin{array}{l}2339 \text { (s.d. of } \\
\operatorname{logs}=3.37 \\
\mathrm{r}=320- \\
76240)\end{array}$ \\
\hline $\begin{array}{l}\text { Parasite density at } \\
\text { reappearance of } \\
\text { parasitaemia } \\
(\text { per } \mu \mathrm{L})\end{array}$ & $\begin{array}{l}1316.9 \text { (s.d. of } \\
\operatorname{logs}=3.12, \\
r=40-52160)\end{array}$ & \\
\hline
\end{tabular}

$\mathrm{CQ}=$ chloroquine, $\mathrm{r}=$ range, s.d. $=$ Standard deviation

The geometric mean of parasitaemia of chloroquine resistant $P$. falciparum primary infections $(5376.7 / \mu \mathrm{L})$ was significantly higher than the recrudescent infections $(1316.9 / \mu \mathrm{L})(\mathrm{t}=3.33, \mathrm{p}<0.01,95 \%$ CI $1.86-8.91)$. Moreover, the mean axillary temperature and the clinical score (16.5) were also significantly higher than the recrudescent infections $(6.6)(\mathrm{t}=6.43, \mathrm{p}<0.001,95 \% \mathrm{CI}$ $1.24-2.36$ and $\mathrm{t}=6.11, \mathrm{p}<0.001,95 \%$ CI $6.68-13.12$ ).

In 26 in vitro assays performed, 23 (88.5\%) were successful; 15 (65.2\%) of the successful assays showed evidence of chloroquine resistance. The distribution of the percentage of schizont maturation inhibition in relation to different chloroquine concentrations is given in Table 2. When compared with in vivo results, there were five infections that showed evidence of chloroquine resistance in vitro only. The mean clinical score and geometric mean of duration of stay, number of previous malaria episodes and parasite density of these five infections were 13.2, 29.7 months, 3.6 and $4746.8 / \mu \mathrm{L}$. The mean clinical score and geometric mean of parasite densities of the five infections were lower than the respective mean figures of in vivo chloroquine resistant $P$. falciparum primary infections and the figures for duration of stay and number of previous malaria attacks were higher than those of the in vivo chloroquine resistant $P$. falciparum group indicating a likelihood of higher level of immunity against malaria in the five patients.

Table 2. Percentage of schizont maturation inhibition at different chloroqine concentrations

\begin{tabular}{cc}
\hline $\begin{array}{c}\text { Chloroquine concentration } \\
(\text { pmol })\end{array}$ & $\begin{array}{r}\text { Schizont maturation } \\
\text { inhibition (\%) }\end{array}$ \\
\hline 0 & 0 \\
1 & 20 \\
2 & 36.6 \\
4 & 56.5 \\
8 & 73.8 \\
16 & 89.9 \\
32 & 99.8 \\
64 & 100 \\
\hline
\end{tabular}

Of 137 patients enrolled for this study, 85 (62\%) claimed that they were on weekly prophylactic chloroquine. This included 69 with $P$. falciparum infections and 16 with $P$. vivax infections; $51(60 \%)$ of those on prophylactic chloroquine had not taken it on a regular basis. There was no significant difference between chloroquine resistant and chloroquine sensitive infections ( $>0.50)$ regarding the regularity of chemoprophylaxis.

\section{Discussion}

We report for the first time in Sri Lanka, the high prevalence of malaria and chloroquine resistance among security forces personnel in an operational area in the Northern Province. This is also the first report on the 
situation of malarial and antimalarial resistance in the Mannar district, one of the five districts of Northern Province, from where no proper data has so far been reported [12-14].

The inversion of the normal pattern of species distribution was a striking finding. Contrary to the usual predominance of $P$. vivax in Sri Lanka, $P$. falciparum has become the predominant species in this study area. More than $50 \%$ of P. falciparum isolates showed some degree of resistance to chloroquine in vivo, and an even higher proportion showed resistance in vitro. Since many of the study population had been exposed to malaria for several years in this area, it is possible that they may have acquired a certain degree of immunity. Thus, the fact that the response of an individual to antimalarials depends on the pharmacodynamic and pharmacokinetic properties of the drug as well as the individual's level of immunity to infection may explain the difference between in vivo and in vitro assays [15].

It has been shown that the selection of drug resistant parasite strains takes place with inappropriate and continuous use of a particular drug in a disease endemic area $[4,16]$, resulting in predominance of the selected isolates. In our study area we found that many patients were diagnosed on clinical suspicion and repeatedly treated with chloroquine, in the absence of diagnostic and follow up facilities. Many were taking prophylactic chloroquine on an irregular basis leading to maintenance of sub-therapeutic levels of chloroquine in infected individuals. This facilitates the selection and the emergence of chloroquine-resistant strains. The vector control efforts and malaria surveillance have also become inefficient due to lack of collaboration between security forces and the local authorities, resulting in an enhanced transmission of selected isolates and underestimation of the true prevalence of malaria in this area. These factors may explain the predominance of $P$. falciparum and high prevalence of chloroquine resistance. With the signing of a Memorandum of Understanding between the Government of Sri Lanka and the Liberation Tigers of Tamil Eelam (LTTE) in February 2002, movement of people between the Northern Province and the rest of the country has increased, and therefore, the risk of transmission of chloroquine resistant strains to other parts of the island is increasing.

Our results indicate that the treatment failure rate with chloroquine in this area was higher than the most recommended levels for change of first-line antimalarial treatment [17-20]. A previous study has also shown that the level of chloroquine resistance in a different geographical setting was as much as 55\% [4]. Hence, we believe that there is an urgent need for revision of the current firstline antimalarial policy in Sri Lanka. It may be necessary to conduct studies in other parts of the country to confirm the geographical extent of chloroquine resistance, but because of the time taken to formulate and implement new national policies, current policy regarding the use of chloroquine in the Northern Province alone, may need more immediate revision.

\section{Acknowledgements}

We thank the Department of Police, particularly the medical assistants and the staff at the Sector 8 Base Camp for providing us support to do this project. We are also thankful to 1st Battalion of Sri Lanka Light Infantry, especially the officers and the soldiers attached to the Pulliadiirakkam Base Camp for their assistance. We extend our sincere gratitude to Regional Malaria Officer and Deputy Provincial Director of Health Services, Vavuniya, for helping us initially to establish our field station. We also thank Prof. A S Dissanaike for reading the manuscript. This study was funded by the National Science Foundation, Sri Lanka (research grant SIDA/ 2001/BT/01).

\section{References}

1. Ratnapala R, Subramaniam K, Yapabandara MGM, Fernando WP. Chloroquine resistant Plasmodium falciparum in Sri Lanka. Sri Lanka Medical Journal 1984; 29: $135-45$.

2. Ministry of Health. Annual Health Bulletin 2000. Colombo, Sri Lanka: Medical Statistics Unit, 2000.

3. Ministry of Health. Annual Health Bulletin 2001. Colombo, Sri Lanka: Medical Statistics Unit, 2001.

4. Handunnetti SM, Gunewardena DM, Pathirana PPSL, Ekanayake K, Weerasinghe S, et al. Features of recrudescent chloroquine-resistant Plasmodium falciparum infections confer a survival advantage on parasites and have implications for disease control. Transactions of the Royal Society of Tropical Medicine and Hygiene 1996; 90: $563-7$.

5. Handunnetti SM, Jayasinghe S, Pathirana PPSL, Fernando $\mathrm{R}$, Sheriff MHR, et al. Sulphadoxine-pyrimethamine and chloroquine resistant Plasmodium falciparum infection in Sri Lanka. Ceylon Medical Journal 1994; 39: 45-6.

6. Gunasekare DPS, Perera DPER. Multidrug resistant malaria in Sri Lanka. Ceylon Medical Journal 1996; 41: $170-1$.

7. Severe falciparum malaria. World Health Organization, Communicable Disease cluster. Transactions of Royal Society of Tropical Medicine and Hygiene 2000; 94: (Suppl. 1): S1-S90.

8. Karunaweera ND, Carter R, Grau GE, Mendis KN Demonstration of anti-disease immunity to Plasmodium vivax malaria in Sri Lanka using a quantitative method to assess clinical disease. American Journal of Tropical Medicine and Hygiene 1998; 58: 204-10.

9. Wernsdorfer WH, Payne D. Drug sensitivity tests in malaria parasites. In: Wernsdorfer WH, McGregor I, eds. Malaria: Principles and Practice of Malariology. 2nd edn. Vol. 2. London: Churchill Livingstone, 1988: 1788-1805. 
10. World Health Organization. Antimalarial drug policies: data requirements, treatment of uncomplicated malaria, and management of malaria in pregnancy. Geneva, Switzerland, 1994. WHO/MAL/94.1070.

11. World Health Organization. In vitro micro-test (mark II) for the assessment of the response of Plasmodium falciparum to chloroquine, mefloquine, quinine, sulfadoxine/pyrimethamine and amodiaquine. Geneva, Switzerland, 1990. WHO/ MAP/87.2 Revision 1.

12. Ministry of Health. Weekly Epidemiological Report 1998. Colombo, Sri Lanka, 1998.

13. Ministry of Health. Weekly Epidemiological Report 1999. Colombo, Sri Lanka, 1999.

14. Ministry of Health. Weekly Epidemiological Report 2000. Colombo, Sri Lanka, 2000.

15. Ringwald $\mathrm{P}$, Basco LK. Comparison of in vivo and in vitro tests of resistance in patients treated with chloroquine in
Yaounde, Cameroon. Bulletin of the World Health Organization 1999; 77: 34-43.

16. Wernsdorfer WH. The development and spread of drug resistant malaria. Parasitology Today 1991; 7: 297-303.

17. World Health Organization. The use of antimalarial drugs: Report of an informal consultation. Geneva, Switzerland, 2001.

18. Bloland PB, Ettling M. Making malaria-treatment policy in the face of drug resistance. Annals of Tropical Medicine and Parasitology 1999; 93: 5-23.

19. World Health Organization. Framework for developing, implementing, and updating antimalarial drug policy in Africa. Malaria, Liaison Bulletin of the Malaria Programme WHO/AFRO 1999; 2: 1-4.

20. Kitua AY. Antimalarial drug policy: making systematic change. Lancet 1999; 354 (Suppl. IV): 32. 